Portland State University

PDXScholar

Electrical and Computer Engineering Faculty

Publications and Presentations

10-1-1982

\title{
Coherence effects in synchronously pumped mode-locked dye lasers
}

Lee W. Casperson

Portland State University

Follow this and additional works at: https://pdxscholar.library.pdx.edu/ece_fac

Part of the Electrical and Computer Engineering Commons

Let us know how access to this document benefits you.

Citation Details

Casperson, L. W. (1982). Coherence effects in synchronously pumped mode-locked dye lasers. Journal of Applied Physics, 54 (5) 2198-2208.

This Article is brought to you for free and open access. It has been accepted for inclusion in Electrical and Computer Engineering Faculty Publications and Presentations by an authorized administrator of PDXScholar. Please contact us if we can make this document more accessible: pdxscholar@pdx.edu. 


\title{
Coherence effects in synchronously pumped mode-locked dye lasers
}

\author{
Lee W. Casperson \\ School of Engineering and Applied Science, University of California, Los Angeles, California 90024
}

(Received 17 August 1982; accepted for publication 5 October 1982)

\begin{abstract}
A formalism is developed for describing the evolution of picosecond mode-locked pulses in synchronously pumped dye lasers. The finite phase memory of the molecular wave functions is included, and it is found that the resultant coherence effects can be dominant for operation close to exact synchrony. Considerations of the isotropic molecular orientational distribution and the finite vibrational relaxation times are also essential in obtaining quantitative agreement with experimental results.
\end{abstract}

PACS numbers: $42.55 . \mathrm{Mv}, 42.65 . \mathrm{Gv}, 42.60 . \mathrm{He}, 42.60 . \mathrm{By}$

\section{INTRODUCTION}

Mode-locked dye lasers are now widely used as sources for tunable intense picosecond pulses of light. The practical applications for such pulses are well known, and one of the most familiar uses is in high-speed time-resolved spectroscopy. When periodic picosecond pulses are required, a standard technique involves synchronous pumping of the dye laser medium using as the pump source a mode-locked argon laser. ${ }^{1-4}$ Typically, the argon laser is mode-locked acoustooptically, and the length of the dye laser cavity is adjusted to be almost exactly equal to the argon laser cavity length (or a multiple thereof). In operation, the dye laser pulse arrives at the dye jet approximately simultaneously with the pump pulse, and this arrangement is found to favor mode-locking of the dye laser. A typical experimental setup is shown schematically in Fig. 1.

To date there have been several rate equation analyses of the synchronously pumped mode-locking process. ${ }^{5-9}$ In some cases, these analyses have provided explicit results for the pulse shapes as functions of the various laser parameters. One of the basic features of all experiments and theories is that the lengths of the argon and dye lasers must be matched very closely to obtain optimum performance. Beyond that, there is, as should be expected, some nonuniformity among the various results; and no previous model has provided more than qualitative indications of the actual pulsation waveforms. In this paper, we present the conclusions of a detailed investigation into the evolution of mode-locked dye laser pulses in a synchronously pumped system. The starting point for this study is a general Maxwell-Schrödinger equation set governing the interaction of the pump and signal fields with the dye laser medium. This model has several advantages over the more conventional rate equation discussions.

First, it may be noted that in the semiclassical MaxwellSchrödinger model, the electric fields are vectors and one can acount in a rigorous way for the fact that the emission and absorption dipoles of the isotropically distributed dye molecules are not generally parallel to the pump and signal fields. The inclusion of an isotropic orientational distribution leads to a quantitative change in the predictions of the model. For practical configurations of synchronously pumped laser systems, however, many qualitative features are the same as for similar models involving unidirectional molecular distributions.

A second feature of the semiclassical approach employed here is that it includes explicitly the nonzero phase memory time of the molecular wave functions. Since this memory time is known to be in the range of $10^{-14}$ to $10^{-13}$ $\mathrm{sec}$, at least for rhodamine-based lasers, it would seem reasonable to expect that the finite phase memory could have at most a minor effect on the evolution of picosecond modelocked pulsations. On the contrary, however, it is shown here that inclusion of phase memory effects is essential to understanding the dye laser dynamics when the length mismatch is very small. For larger detunings, on the other hand, the phase memory time can be approximated by zero.

Another feature of the model developed here is that it includes a finite vibrational relaxation time in the lower electronic state of the dye laser transition. The vibrational relaxation time is on the order of one picosecond, and the inclusion of this effect is shown to be of great importance in interpreting the evolution of the picosecond laser pulses.

The pulsation behavior of synchronously pumped lasers is marvelously complicated and a substantial amount of experimental data has been published. Generally, the dye laser pulses are found to have a complex structure which is highly repeatable from pulse to pulse, but which varies dramatically with conditions of gain, loss, and cavity length mismatch. This wealth of data is an advantage for purposes of modelling, but it is also clear that only a reasonably correct model will be able to handle this wide range of experimental conditions without many adjustable parameters. In Sec. II, the theoretical model used here is developed from the basic Maxwell-Schrödinger equations, including the features mentioned in the previous paragraphs. In Sec. III, the best available data concerning pulse shapes and parameter values in a synchronously pumped rhodamine $6 \mathrm{G}$ dye laser are discussed and summarized. These data are then com-

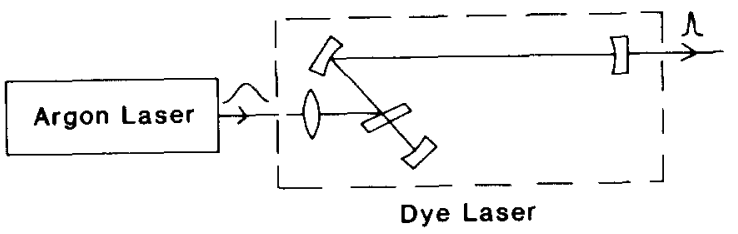

FIG. 1. Schematic representation of a typical synchronously pumped mode-locked dye laser setup. 
pared in Sec. IV with the detailed predictions of the theoretical formulation. After investigating the validity of the general model, various simplifications are considered to rigorously test the importance of including an isotropic molecular distribution, a finite vibrational relaxation time, or a finite phase coherence time. It is concluded that none of these effects can be ignored if one wishes to obtain quantitative agreement between theory and experiment.

\section{SEMICLASSICAL MODEL}

Since the work of Lamb and others, the MaxwellSchrödinger semiclassical model has become well established as a reasonable starting point in any laser calculations where rapid fluctuations of the fields or populations are anticipated or where the vector nature of the fields is expected to be important. ${ }^{10}$ In this section, a specialized semiclassical model is developed for treating the evolution of mode-locked pulsations in a synchronously pumped dye laser oscillator. The model is simplified as much as possible without losing any terms that are expected to play an important role in the laser dynamics on a picosecond time scale.

\section{A. Density matrix equations}

The energy level model used in the treatment is shown schematically in Fig. 2. This is the same as the model used recently in a study of polarization effects in dye laser amplifiers, and much of the notation employed here is also the same. ${ }^{11,12}$ The dye molecules in this model are assumed to be representable by a four-level system in which the pump absorption takes place between Levels 0 and 3 , while the signalstimulated emission takes place between Levels 2 and 1 . The molecules in Level 3 decay nonradiatively to Level 2 with a vibrational relaxation time $\tau_{3}$, while molecules in Level 1 have a vibrational relaxation time of $\tau_{1}$. The spontaneous decay time for the laser transition is represented by $\tau_{2}$. The actual energy level structure of the dye molecules is more complex than this simple model would indicate, but the model does at least include the essential features of vibrational relaxation into and out of the laser levels. Also, for the effects of interest here, intersystem crossing to the triplet states simply leads to a reduction in the pump efficiency, which does not need to be explicitly included in the analysis.

The procedure for setting up the density matrix equations is well known, and for the present energy level model, the results are ${ }^{11}$ :

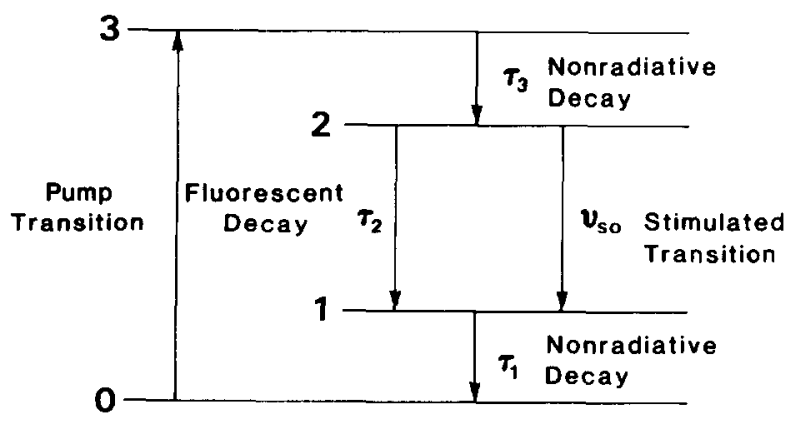

FIG. 2. Energy level model used in the dye laser analysis.

$$
\begin{aligned}
& \frac{\partial \rho_{00}}{\partial t}=-\frac{i}{\hbar}\left(\rho_{03} \boldsymbol{\mu}_{30}-\rho_{30} \boldsymbol{\mu}_{03}\right) \cdot \mathbf{E}_{p}+\frac{\rho_{11}}{\tau_{1}} \\
& \frac{\partial \rho_{33}}{\partial t}=\frac{i}{\hbar}\left(\rho_{03} \boldsymbol{\mu}_{30}-\rho_{30} \boldsymbol{\mu}_{03}\right) \cdot \mathbf{E}_{p}-\frac{\rho_{33}}{\tau_{3}} \\
& \frac{\partial \rho_{22}}{\partial t}=-\frac{i}{\hbar}\left(\rho_{21} \boldsymbol{\mu}_{12}-\rho_{12} \boldsymbol{\mu}_{21}\right) \cdot \mathbf{E}_{s}-\frac{\rho_{22}}{\tau_{2}}+\frac{\rho_{33}}{\tau_{3}}, \\
& \frac{\partial \rho_{11}}{\partial t}=\frac{i}{\hbar}\left(\rho_{21} \boldsymbol{\mu}_{12}-\rho_{12} \boldsymbol{\mu}_{21}\right) \cdot \mathbf{E}_{s}-\frac{\rho_{11}}{\tau_{1}}+\frac{\rho_{22}}{\tau_{2}}, \\
& \frac{\partial \rho_{30}}{\partial t}=-i \omega_{p 0} \rho_{30}+\frac{i}{\hbar}\left(\rho_{00}-\rho_{33}\right) \boldsymbol{\mu}_{30} \cdot \mathbf{E}_{p}-\frac{\rho_{30}}{T_{p}} \\
& \frac{\partial \rho_{21}}{\partial t}=-i \omega_{s o} \rho_{21}-\frac{i}{\hbar}\left(\rho_{22}-\rho_{11}\right) \boldsymbol{\mu}_{21} \cdot \mathbf{E}_{s}-\frac{\rho_{21}}{T_{s}} \\
& \rho_{03}=\rho_{30}^{*}, \\
& \rho_{12}=\rho_{21}^{*},
\end{aligned}
$$

where $\mathbf{E}_{p}$ and $\mathbf{E}_{s}$ are, respectively, the pump and signal electric fields, $\omega_{p o}$ and $\omega_{s o}$ are, respectively, the center frequencies of the pump and signal transitions, the various $\mu_{j i}$ are the matrix elements of the dipole moment operator, and $T_{p}$ and $T_{s}$ are the coherence times of the off-diagonal matrix elements. It is convenient to multiply Eqs. (5) and (6) by $\mu_{03}$ and $\mu_{12}$ respectively, and the results are

$$
\begin{aligned}
& \frac{\partial \boldsymbol{\eta}_{30}}{\partial t}=-i \omega_{p 0} \boldsymbol{\eta}_{30}+\frac{i}{\hbar}\left(\rho_{00}-\rho_{33}\right)\left|\mu_{p}\right|^{2}\left(\mathbf{E}_{p} \cdot \hat{e}_{p}\right) \hat{e}_{p}-\frac{\boldsymbol{\eta}_{30}}{T_{p}}, \\
& \frac{\partial \boldsymbol{\eta}_{21}}{\partial t}=-i \omega_{s 0} \boldsymbol{\eta}_{21}-\frac{i}{\hbar}\left(\rho_{22}-\rho_{11}\right)\left|\mu_{s}\right|^{2}\left(\mathbf{E}_{s} \cdot \hat{e}_{s}\right) \hat{e}_{s}-\frac{\eta_{21}}{T_{s}},
\end{aligned}
$$

where the new parameters are

$$
\begin{aligned}
& \eta_{30}=\rho_{30} \mu_{03}=\rho_{30} \mu_{03} \hat{e}_{p}, \\
& \eta_{21}=\rho_{21} \mu_{12}=\rho_{21} \mu_{12} \hat{e}_{s}, \\
& \left|\mu_{p}\right|^{2}=\mu_{30} \mu_{30}^{*}=\mu_{30} \mu_{03}, \\
& \left|\mu_{s}\right|^{2}=\mu_{21} \mu_{21}^{*}=\mu_{21} \mu_{12},
\end{aligned}
$$

and $\hat{e}_{p}$ and $\hat{e}_{s}$ are unit vectors in the directions of $\boldsymbol{\mu}_{03}$ and $\boldsymbol{\mu}_{12}$, respectively.

Equations (1)-(4) and (9) and (10) describe the response of the dye laser medium to an arbitrary electric field. These equations can be simplified substantially for the case of a synchronously pumped mode-locked dye laser by specializing to the case of plane-wave pump and signal fields having parallel propagation vectors $\mathbf{k}_{p}$ and $\mathbf{k}_{s}$. For propagation in the $z$ direction, the field components can be written

$$
\begin{aligned}
& \mathbf{E}_{p}(z, t)=\frac{1}{2} \mathscr{E}_{p}(z, t) \exp \left[i\left(k_{p} z-\omega_{p} t\right)\right]+\text { c.c., } \\
& \mathbf{E}_{s}(z, t)=\frac{1}{2} \mathscr{E}_{s}(z, t) \exp \left[i\left(k_{s} z-\omega_{s} t\right)\right]+\text { c.c., }
\end{aligned}
$$

where $\mathscr{E}_{p}(z, t)$ and $\mathscr{E}_{s}(z, t)$ are slowly varying complex vector functions of $z$ and $t$. This form for the electric fields is rigorously valid for a ring dye laser, but many of the results described below should apply also in standing-wave lasers if the thin active medium is close to one of the resonator mirrors. The plane-wave dependence in the off-diagonal matrix elements can be factored out using the substitutions

$$
\eta_{30}(\theta, \phi, z, t)=\eta_{p}(\theta, \phi, z, t) \exp \left[i\left(k_{p} z-\omega_{p} t\right)\right] \hat{e}_{p},
$$




$$
\eta_{21}(\theta, \phi, z, t)=\eta_{s}(\theta, \phi, z, t) \exp \left[i\left(k_{s} z-\omega_{s} t\right)\right] \hat{e}_{s},
$$

where in Eq. (17), $\theta$ measures the angle of a class of pump dipoles with respect to the $x$ axis (which is perpendicular to the propagation direction), and $\phi$ measures the orientation of the dipoles around the $x$ axis. ${ }^{11}$ A similar interpretation applies to the signal dipoles described in Eq. (18). With Eqs. (15)-(18) together with the rotating-wave approximation, Eqs. (1)-(4) and (9) and (10) reduce to

$$
\begin{aligned}
& \frac{\partial \rho_{00}}{\partial t}=-\frac{1}{\hbar} \operatorname{Im}\left(\boldsymbol{\eta}_{p} \cdot \mathscr{E}_{p}^{*}\right)+\frac{\rho_{11}}{\tau_{1}} \\
& \frac{\partial \rho_{33}}{\partial t}=\frac{1}{\hbar} \operatorname{Im}\left(\boldsymbol{\eta}_{p} \cdot \mathscr{E}_{p}^{*}\right)-\frac{\rho_{33}}{\tau_{3}} \\
& \frac{\partial \rho_{22}}{\partial t}=\frac{1}{\hbar} \operatorname{Im}\left(\boldsymbol{\eta}_{s} \cdot \mathscr{E}_{s}^{*}\right)-\frac{\rho_{22}}{\tau_{2}}+\frac{\rho_{33}}{\tau_{3}}, \\
& \frac{\partial \rho_{11}}{\partial t}=-\frac{1}{\hbar} \operatorname{Im}\left(\boldsymbol{\eta}_{s} \cdot \mathscr{C}_{s}^{*}\right)-\frac{\rho_{11}}{\tau_{1}}+\frac{\rho_{22}}{\tau_{2}} \\
& \frac{\partial \eta_{p}}{\partial t} \\
&=i\left(\omega_{p}-\omega_{p o}\right) \eta_{p}+\frac{i}{2 \hbar}\left(\rho_{00}-\rho_{33}\right)\left|\mu_{p}\right|^{2} \mathscr{E}_{p} \cdot \hat{e}_{p}-\frac{\eta_{p}}{T_{p}} \\
& \frac{\partial \eta_{s}}{\partial t} \\
&=i\left(\omega_{s}-\omega_{s o}\right) \eta_{s}-\frac{i}{2 \hbar}\left(\rho_{22}-\rho_{11}\right)\left|\mu_{s}\right|^{2} \mathscr{E}_{s} \cdot \hat{e}_{s}-\frac{\eta_{s}}{T_{s}},
\end{aligned}
$$

where $\boldsymbol{\eta}_{p}=\eta_{p} \hat{e}_{p}$ and $\eta_{s}=\eta_{s} \hat{e}_{s}$.

The average value of the polarization induced by the pump and stimulating fields is given by

$$
\begin{aligned}
& \mathbf{P}_{\rho}=\int_{\Omega} n(\theta, \phi)\left(\rho_{03} \mu_{30}+\rho_{30} \mu_{03}\right) d \Omega, \\
& \mathbf{P}_{s}=\int_{\Omega} n(\theta, \phi)\left(\rho_{21} \boldsymbol{\mu}_{12}+\rho_{12} \mu_{21}\right) d \Omega,
\end{aligned}
$$

where $n(\theta, \phi) d \Omega$ is the number of molecules per unit volume oriented within the solid angle $d \Omega$ about the $(\theta, \phi)$ direction. With the previous definitions, these polarizations can be written

$$
\begin{aligned}
& \mathbf{P}_{p}=\int_{\Omega} n(\theta, \phi) \eta_{p}(\theta, \phi, z, t) \hat{e}_{p} d \Omega \exp \left[i\left(k_{p} z-\omega_{p} t\right)\right]+\text { c.c. } \\
& \mathbf{P}_{s}=\int_{\Omega} n(\theta, \phi) \eta_{s}(\theta, \phi, z, t) \hat{e}_{s} d \Omega \exp \left[i\left(k_{s} z-\omega_{s} t\right)\right]+\text { c.c. }
\end{aligned}
$$

Now Eqs. (19)-(24) and (27) and (28) may be taken as a complete set implying the pump and signal polarization components in terms of the pump and signal electric fields. The next step in this analysis is to develop equations which express the fields in terms of the polarizations.

\section{B. Maxwell's equations}

From Maxwell's equations, the pump and signal vectors are related to the corresponding polarizations by the wave equations

$$
\begin{aligned}
& \frac{\partial^{2} \mathbf{E}_{p}}{\partial z^{2}}-\mu \epsilon \frac{\partial^{2} \mathbf{E}_{p}}{\partial t^{2}}-\mu \sigma \frac{\partial \mathbf{E}_{p}}{\partial t}=\mu \frac{\partial^{2} \mathbf{P}_{p}}{\partial t^{2}}, \\
& \frac{\partial^{2} \mathbf{E}_{s}}{\partial z^{2}}-\mu \epsilon \frac{\partial^{2} \mathbf{E}_{s}}{\partial t^{2}}-\mu \sigma \frac{\partial \mathbf{E}_{s}}{\partial t}=\mu \frac{\partial^{2} \mathbf{P}_{s}}{\partial t^{2}} .
\end{aligned}
$$

Using Eqs. (15), (16), (25), and (26), these wave equations reduce to

$$
\begin{aligned}
& \frac{\partial \mathscr{E}_{p}}{\partial z}+\frac{1}{v} \frac{\partial \mathscr{E}_{p}}{\partial t}+\frac{\gamma_{p}}{2} \mathscr{E}_{p}=i \mu \frac{\omega_{p}^{2}}{k_{p}} \int_{\Omega} n(\theta, \phi) \eta_{p} \hat{e}_{p} d \Omega \\
& \frac{\partial \mathscr{E}_{s}}{\partial z}+\frac{1}{v} \frac{\partial \mathscr{E}_{s}}{\partial t}+\frac{\gamma_{s}}{2} \mathscr{C}_{s}=i \mu \frac{\omega_{s}^{2}}{k_{s}} \int_{\Omega} n(\theta, \phi) \eta_{s} \hat{e}_{s} d \Omega
\end{aligned}
$$

where higher order space and time derivatives of slowly varying quantities have been neglected, and the rotating-wave approximation has been employed. Also, the propagation constants are given by $k_{p}^{2}=\mu \epsilon \omega_{p}^{2}, k_{s}^{2}=\mu \epsilon \omega_{s}^{2}$, and the approximate velocity $v=(\mu \epsilon)^{-1 / 2}$ has been introduced.

\section{Simplifications and specializations}

Equations (19)-(24) and (31) and (32) form a complete set for investigating the interaction of the laser pump and signal fields with an arbitrary dye laser medium. These equations are more general than necessary for the present application; and, as indicated previously, the objective here is to obtain the simplest reasonable model to test against experimental results concerning synchronously pumped modelocked dye lasers. Accordingly, several simplifications will now be introduced.

If the pump and signal frequencies are close to the center frequencies of their respective transitions $\left(\omega_{p} \simeq \omega_{p o}\right.$, $\left.\omega_{s} \simeq \omega_{s o}\right)$, and saturation of the pump transition is weak $\left(1 \simeq \rho_{00} \gg \rho_{33}\right)$, then Eqs. $(23)$ and (24) reduce to

$$
\begin{aligned}
& \frac{\partial \eta_{p}}{\partial t}=\frac{i}{2 \hbar}\left|\mu_{p}\right|^{2} \mathscr{E}_{p} \cdot \hat{e}_{p}-\frac{\eta_{p}}{T_{p}}, \\
& \frac{\partial \eta_{s}}{\partial t}=\frac{i}{2 \hbar}\left(\rho_{22}-\rho_{11}\right)\left|\mu_{s}\right|^{2} \mathscr{E}_{s} \cdot \hat{e}_{s}-\frac{\eta_{s}}{T_{s}} .
\end{aligned}
$$

These approximations would not always be valid, but they seem to provide adequate agreement with available data. If necessary, the more general equations could be retained.

As will be discussed below, practical values for the coherence times $T_{p}$ and $T_{s}$ are on the order of $10^{-13} \mathrm{sec}$. On the other hand, the pump pulses from the argon laser have a width on the order of $10^{-10} \mathrm{sec}$. Therefore, the time derivative in Eq. (33) may be neglected as $\eta_{p}$ tracks instantaneously the value of the pump field amplitude. Thus, Eq. (33) has the solution

$$
\eta_{p}=\frac{i T_{p}}{2 \hbar}\left|\mu_{p}\right|^{2} \mathscr{E}_{p} \cdot \hat{e}_{p} .
$$

The dye laser pulse is much shorter than the pump pulse, and it is not obvious that the corresponding derivative in Eq. (34) could be neglected. In fact, it will be shown below that neglect of this derivative would lead to major errors in the predictions of the theory.

The width of the pump pulse can also be shown to be long compared to the vibrational relaxation times $\tau_{3}$ and $\tau_{1}$. Therefore, the time derivative in Eq. (21) is unimportant, and this equation may be combined with Eq. (35) to obtain the population

$$
\rho_{33}=\frac{\tau_{3} T_{p}}{2 \hbar^{2}}\left|\mu_{p}\right|^{2}\left|\mathscr{E}_{p} \cdot \hat{e}_{p}\right|^{2} .
$$

With this result, Eqs. (21), (22), (32), and (34) may be written

$$
\frac{\partial \rho_{22}}{\partial t}=\frac{1}{\hbar} \operatorname{Im}\left(\boldsymbol{\eta}_{s} \cdot \mathscr{E}_{s}^{*}\right)-\frac{\rho_{22}}{\tau_{2}}+\frac{T_{p}}{2 \hbar^{2}}\left|\mu_{p}\right|^{2}\left|\mathscr{E}_{p} \cdot \hat{e}_{p}\right|^{2},
$$




$$
\begin{aligned}
\frac{\partial \rho_{11}}{\partial t} & =\frac{1}{\hbar} \operatorname{Im}\left(\eta_{s} \cdot \mathscr{E}_{s}^{*}\right)-\frac{\rho_{11}}{\tau_{1}}+\frac{\rho_{22}}{\tau_{2}}, \\
\frac{\partial \eta_{s}}{\partial t} & =-\frac{i}{2 \hbar}\left(\rho_{22}-\rho_{11}\right)\left|\mu_{s}\right|^{2} \mathscr{E}_{s} \cdot \hat{e}_{s}-\frac{\eta_{s}}{T_{s}}, \\
\frac{\partial \mathscr{E}_{s}}{\partial z} & +\frac{1}{v} \frac{\partial \mathscr{C}_{s}}{\partial t}+\frac{\gamma_{s}}{2} \mathscr{E}_{s} \\
& =i \mu \frac{\omega_{s}^{2}}{k_{s}} \int_{\Omega} n(\theta, \phi) \eta_{s} \hat{e}_{s} d \Omega .
\end{aligned}
$$

As long as pump depletion is not too severe, the field $\mathscr{E}_{p}$ may be regarded as a known quantity, and only these four equations are needed for a realistic discussion of a synchronously pumped mode-locked dye laser.

It can now be assumed that $\mathscr{E}_{p}$ and $\mathscr{E}_{s}$ are real vectors, while $\eta_{s}$ is purely imaginary. With the substitution $\eta_{s}=i \eta_{s}^{\prime}$, Eqs. (37)-(40) reduce to the four real equations:

$$
\begin{gathered}
\frac{\partial \rho_{22}}{\partial t}=\frac{1}{\hbar} \eta_{s}^{\prime} \mathscr{E}_{s} \cdot \hat{e}_{s}-\frac{\rho_{22}}{\tau_{22}}+\frac{T_{p}\left|\mu_{p}\right|^{2}}{2 \hbar^{2}}\left(\mathscr{E}_{p} \cdot \hat{e}_{p}\right)^{2},(4) \\
\frac{\partial \rho_{11}}{\partial t}=-\frac{1}{\hbar} \eta_{s}^{\prime} \mathscr{E}_{s} \cdot \hat{e}_{s}-\frac{\rho_{11}}{\tau_{1}}+\frac{\rho_{22}}{\tau_{2}} \\
\frac{\partial \eta_{s}^{\prime}}{\partial t}=-\frac{1}{2 \hbar}\left(\rho_{22}-\rho_{11}\right)\left|\mu_{s}\right|^{2} \mathscr{E}_{s} \cdot \hat{e}_{s}-\frac{\eta_{s}^{\prime}}{T_{s}} \\
\frac{\partial \mathscr{E}_{s}}{\partial z}+\frac{1}{v} \frac{\partial \mathscr{E}_{s}}{\partial t}+\frac{\gamma_{s}}{2} \mathscr{E}_{s}=-\frac{\mu \omega_{s}^{2}}{k_{s}} \int_{\Omega} n(\theta, \phi) \eta_{s}^{\prime} \hat{e}_{s} d \Omega
\end{gathered}
$$

In most dye molecules, the absorption and emission dipoles are parallel $\left(\hat{e}_{p}=\hat{e}_{s}\right)$. Also, in most synchronously pumped mode-locked dye lasers, the pump and signal field polarizations are parallel to each other, and it is assumed here that these fields are parallel to the $x$ axis. Thus, Eqs. (41)-(44) become

$$
\begin{gathered}
\frac{\partial \rho_{22}}{\partial t}=\frac{1}{\hbar} \eta_{s}^{\prime} \mathscr{E}_{s} \cos \theta-\frac{\rho_{22}}{\tau_{2}}+\frac{T_{p}\left|\mu_{p}\right|^{2}}{2 \hbar^{2}} \mathscr{C}_{p}^{2} \cos ^{2} \theta \\
\frac{\partial \rho_{11}}{\partial t}=-\frac{1}{\hbar} \eta_{s}^{\prime} \mathscr{E}_{s} \cos \theta-\frac{\rho_{11}}{\tau_{1}}+\frac{\rho_{22}}{\tau_{2}}, \\
\frac{\partial \eta_{s}^{\prime}}{\partial t}=-\frac{1}{2 \hbar}\left(\rho_{22}-\rho_{11}\right)\left|\mu_{s}\right|^{2} \mathscr{E}_{s} \cos \theta-\frac{\eta_{s}^{\prime}}{T_{s}}, ~(47) \\
\frac{\partial \mathscr{E}_{s}}{\partial z}+\frac{1}{v} \frac{\partial \mathscr{C}_{s}}{\partial t}+\frac{\gamma_{s}}{2} \mathscr{E}_{s} \\
=-\frac{\mu \omega_{s}^{2}}{k_{s}} \int_{\Omega} n(\theta, \phi) \eta_{s}^{\prime} \cos \theta d \Omega
\end{gathered}
$$

where $\mathscr{C}_{s}$ represents the amplitude of the vector electric field $\mathscr{E}_{s}$.

At this point, it is necessary to specify the orientational distribution of the dye molecules in the laser amplifier. In spherical coordinates, the differential solid angle element is $d \Omega=\sin \theta d \theta d \phi$, and the angular distribution of dye molecules in a liquid can usually be written $n(\theta, \phi)=N / 4 \pi$. With these substitutions, Eqs. $(45)-(48)$ can be written

$$
\begin{aligned}
& \frac{\partial \rho_{22}}{\partial t}=\frac{1}{\hbar} \eta_{s}^{\prime} \mathscr{E}_{s} x-\frac{\rho_{22}}{\tau_{2}}+\frac{T_{p}\left|\mu_{p}\right|^{2}}{2 \hbar^{2}} \mathscr{E}_{p}^{2} x^{2} \\
& \frac{\partial \rho_{11}}{\partial t}=-\frac{1}{\hbar} \eta_{s}^{\prime} \mathscr{E}_{s} x-\frac{\rho_{11}}{\tau_{1}}+\frac{\rho_{22}}{\tau_{2}} \\
& \frac{\partial \eta_{s}^{\prime}}{\partial t}=-\frac{1}{2 \hbar}\left(\rho_{22}-\rho_{11}\right)\left|\mu_{s}\right|^{2} \mathscr{E}_{s} x-\frac{\eta_{s}^{\prime}}{T_{s}} \\
& \frac{\partial \mathscr{E}_{s}}{\partial z}+\frac{1}{v} \frac{\partial \mathscr{E}_{s}}{\partial t}+\frac{\gamma_{s}}{2} \mathscr{E}_{s}=-\frac{\mu \omega_{s}^{2} N}{k_{s}} \int_{0}^{1} \eta_{s}^{\prime} x d x
\end{aligned}
$$

where the new variable $x=\cos \theta$ has been introduced, and use has been made of the fact that $\eta_{s}^{\prime}$ is an odd function of $x$.

As a next step, Eqs. (49)-(52) can be written in a more compact form if one introduces suitably normalized forms for the dependent variables. The choices adopted here include

$$
\begin{aligned}
& D=\frac{\mu \omega_{s}^{2} N T_{s}\left|\mu_{s}\right|^{2}}{k_{s} \hbar \gamma}\left(\rho_{22}-\rho_{11}\right), \\
& M=\frac{\mu \omega_{s}^{2} N T_{s}\left|\mu_{s}\right|^{2}}{k_{s} \hbar \gamma}\left(\rho_{22}+\rho_{11}\right), \\
& Q=-\frac{\mu \omega_{s}^{2} N\left|\mu_{s}\right|\left(2 \tau_{2} T_{s}\right)^{1 / 2}}{k_{s} \hbar \gamma} \eta_{s}^{\prime}, \\
& A=\frac{\left|\mu_{s}\right|}{\hbar}\left(\frac{\tau_{2} T_{s}}{2}\right)^{1 / 2} \mathscr{C}_{s}, \\
& P=\frac{\mu \omega_{s}^{2} N T_{s}\left|\mu_{s}\right|^{2}}{k_{s} \hbar \gamma} \frac{\tau_{3} T_{p}\left|\mu_{p}\right|^{2} \mathscr{E}_{p}^{2}}{2 \hbar^{2}} .
\end{aligned}
$$

Thus $D$ is a normalized population difference, $M$ is a normalized population sum, $Q$ is a normalized polarization, $A$ is a normalized field, and $P$ is a normalized pump rate. In terms of these new variables, Eqs. (49)-(52) simplify to

$$
\begin{aligned}
& \frac{\partial D}{\partial t} \\
& =-\frac{1}{\tau_{2}}\left[\left(1+\frac{\tau_{2}}{2 \tau_{1}}\right) D+\left(1-\frac{\tau_{2}}{2 \tau_{1}}\right) M+2 Q A x-P x^{2}\right] \\
& \frac{\partial M}{\partial t}=-\frac{1}{\tau_{2}}\left(-\frac{\tau_{2}}{2 \tau_{1}} D+\frac{\tau_{2}}{2 \tau_{1}} M-P x^{2}\right) \\
& \frac{\partial Q}{\partial t}=-\frac{1}{T_{s}}(Q-A D x) \\
& \frac{\partial A}{\partial z}+\frac{1}{v} \frac{\partial A}{\partial t}=-\frac{\gamma}{2}\left(A-\int_{0}^{1} Q x d x\right)
\end{aligned}
$$

\section{Synchronous pumping}

Equations (58)-(61) may be used to describe the evolution of a short pulse of light in a dye laser amplifier having arbitrary space- and time-dependent pump and loss rates. These equations can be readily adapted to the special problem of a synchronously pumped ring dye laser oscillator. In such a laser, the signal field amplitude $A$ must be periodic with the same period as the pump pulse. If the net gain per pass in the laser amplifier is not too large, each molecule in the dye medium interacts with the same time-dependent 
pump and signal amplitudes. Thus, the output of the dye laser is the same as it would be if the gain and loss were uniformly distributed around the dye cavity with the pump and signal pulses having exactly equal velocities. There is, however, no reason why these velocities should be considered to be equal to the speed of light $v$. The repetition rate of the pump pulse is governed primarily by parameters of the argon laser, and with a saturable gain, it is possible for the effective velocity of the dye laser signal to be somewhat greater than or less than the speed of light. To take advantage of these concepts, it is helpful to introduce a new time coordinate $\tau=t-z / v_{s}$, where $v_{s}$ is the "envelope speed" in the dye laser of all quantities of interest. Then Eqs. (58)-(61) reduce to the ordinary differential equations

$$
\begin{aligned}
& \frac{d D}{d \tau} \\
& =-\frac{1}{\tau_{2}}\left[\left(1+\frac{\tau_{2}}{2 \tau_{1}}\right) D+\left(1-\frac{\tau_{2}}{2 \tau_{1}}\right) M+2 Q A x-P x^{2}\right], \\
& \frac{d M}{d \tau}=-\frac{1}{\tau_{2}}\left(-\frac{\tau_{2}}{2 \tau_{1}} D+\frac{\tau_{2}}{2 \tau_{1}} M-P x^{2}\right), \\
& \frac{d Q}{d \tau}=-\frac{1}{T_{s}}(Q-A D x), \\
& \frac{d A}{d \tau}=-\frac{1}{2 t_{c}\left(1-v / v_{s}\right)}\left(A-\int_{0}^{1} Q x d x\right),
\end{aligned}
$$

where all of the cavity losses are now incorporated in the usual photon-cavity lifetime. Equations (62)-(65) constitute a fairly general set which will be used in Sec. IV to calculate the response of a dye laser driven by periodic pump pulses.

\section{DATA}

As indicated previously, synchronously pumped modelocked dye lasers are now in wide use and have found many practical applications. Commercial systems are available, and much experimental data have been published. Some of the published data is well documented and can be used to objectively test the validity of the model that has been developed here. In this section, a particular set of pulsation waveforms is examined and values for the various dye and cavity parameters are deduced. In the following section, these data are used in studying the consequences of the theoretical model. An example of a set of pulsation waveforms is given in Fig. 3. ${ }^{8}$ These autocorrelation traces were obtained using an experimental setup of the type indicated in Fig. 1. The number labeling each trace is the relative length of the dye cavity in microns, and the absolute cavity length must be inferred from other considerations. The curves shown in Fig. 3 include the most important operating region for a synchronously pumped mode-locked dye laser. In particular, for relative cavity lengths of above 510 microns, it is clear from the figure that the output consists of a single pulse which is both short and intense. In fact, it is just the need for pulses with these characteristics that has provided the motivation for the development of synchronously pumped mode-locked dye lasers. For relative cavity lengths much longer than 510

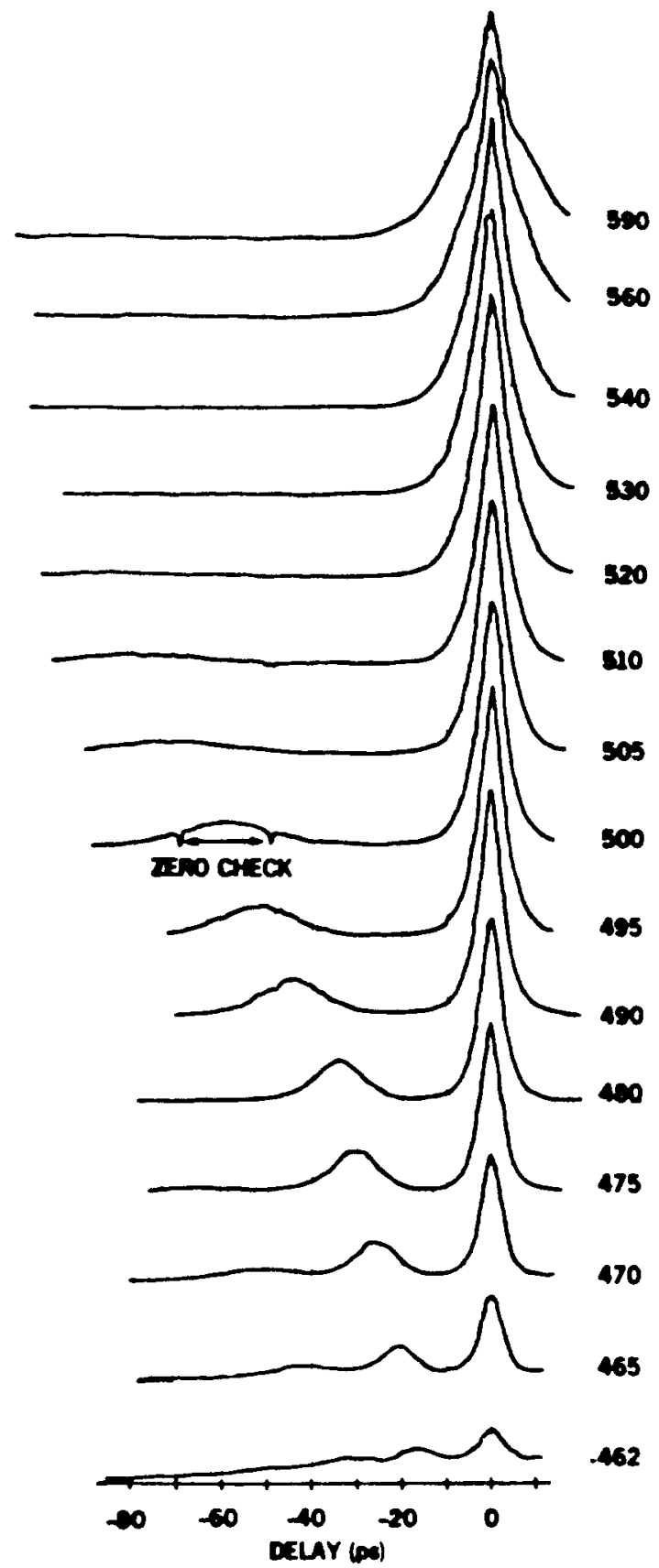

FIG. 3. Autocorrelation traces of pulses obtained from a synchronously pumped mode-locked dye laser (after Ref. 8). The numbers on the side indicate the relative cavity length detuning $\Delta L$ in microns.

microns, the pulses weaken and the additional structure on the autocorrelation traces suggests the occurrence of undesirable echo pulses. The purpose of the next section is to see whether the general theoretical model that has been developed here is able to reproduce the essential features of the data given in Fig. 3.

As exemplified by Fig. 3, one of the most important measurable parameters in a study of synchronous modelocking is the relative length of the dye laser cavity. For this reason, it is convenient in Eq. (65) to express the velocities in terms of the lengths of the argon and dye laser cavities. Thus, the pulse speed in the dye cavity can be related to the round 
trip time by $v_{s}=L_{\mathrm{dye}} / t_{r t}$. On the other hand, the round trip time is given by $t_{r t}=L_{\text {arg }} / v_{s}^{\prime}$, where $v_{s}^{\prime}$ is the effective pulse speed in the argon laser cavity. If one defines an effective round trip argon cavity length $L_{\text {arg }}^{\prime}=L_{\text {arg }} v / v_{s}^{\prime}$, then the needed velocity ratio is simply $v / v_{s}=L_{\text {arg }}^{\prime} / L_{\text {dye }}$. If one further notes that the length difference $\Delta L=L_{\mathrm{dye}}-L_{\text {arg }}^{\prime}$ is much smaller than $L$ arg $\simeq L_{\mathrm{dye}} \equiv L$, then Eq. (65) can be written

$$
\frac{d A}{d \tau}=-\frac{L}{2 t_{c} \Delta L}\left(A-\int_{0}^{1} Q x d x\right) .
$$

To test the model developed here, one may compare numerical solutions of Eqs. (62)-(64) and (66) to the experimental results shown in Fig. 3. First, however, it is necessary to obtain values for the various coefficients appearing in these equations. The fluorescence decay time of rhodamine 6G is well known; and, as in other treatments, a value of $\tau_{2}=5 \times 10^{-9} \mathrm{sec}$ is employed here. ${ }^{6,8}$ The vibrational relaxation time is much shorter and not quite so precisely known. Some studies have indicated that the vibrational relaxation times in both upper and lower electronic states of Rhodamine $6 \mathrm{G}$ are about $(3 \pm 2) \times 10^{-12} \mathrm{sec}^{13,14}$ Other research has shown that the vibrational relaxation time may be somewhat shorter (at least in the upper electronic state $S_{1}$ ), and a relaxation time of $(0.8 \pm 0.2) \times 10^{-12} \mathrm{sec}$ has been deduced. ${ }^{15}$ For the present study, the approximate compromise value $\tau_{1}=1 \times 10^{-12} \mathrm{sec}$ has been adopted.

The coherence time $T_{s}$ for rhodamine $6 \mathrm{G}$ is shorter still, and its value is not yet precisely known. The coherence times for several dyes, including rhodamine $6 \mathrm{G}$, have all been found to lie in the range of $0.02 \times 10^{-12} \mathrm{sec}$ to $2.0 \times 10^{-12} \mathrm{sec}$, depending on solvents, spectral positions, and sample deterioration. ${ }^{16}$ More specific information is presently available for only a few dyes. A lower bound on the coherence time for rhodamine $6 \mathrm{G}$ can be obtained from measurements of the time-resolved fluorescence spectrum, and such measurements have shown that immediately after a short pump pulse, the fluorescence is a Lorentzian function having a full width at half maximum of $12 \mathrm{~nm} .{ }^{17}$ With pure homogeneous broadening, this would correspond to a homogeneous linewidth of $\Delta v_{h}=1.15 \times 10^{13} \mathrm{~Hz}$ and a minimum coherence time of $2.8 \times 10^{-14} \mathrm{sec}$. Given the limitations of the available data, it was decided here to simply adopt the previously inferred value $T_{s}=5 \times 10^{-14} \mathrm{sec}^{18,19}$

In principle, the cavity lifetime $t_{c}$ can be determined by considering the various losses in the laser. In the present case, however, these losses are not known well enough to allow more than an approximate estimate of $t_{c}$. According to the description of the experiment associated with Fig. 3, the intensity transmission is $T=46 \%$ (Ref. 8). In addition, it can be anticipated that there would be substantial losses associated with the intracavity birefringent filters, other surfaces in the cavity, and absorption and scattering in the dye itself. All that can be said in advance is that the cavity lifetime should be on the order of several nsec, and in many of our computations, a value $t_{c}=4 \times 10^{-9} \mathrm{sec}$ has been adopted.

As a final preliminary, it is necessary to specify the pump function $P(\tau)$. In the experiment, the pumping signal is approximately a Gaussian of width 100 psec (Ref. 8). Thus, the pump function can be written $P(\tau)=P_{0} f(\tau)$, where $f(\tau)$ is the normalized function

$$
f(\tau)=\frac{2}{\Delta \tau}\left(\frac{\ln 2}{\pi}\right)^{1 / 2} \exp \left[-\left(\frac{2 \tau}{\Delta \tau}\right)^{2} \ln 2\right] .
$$

The full width at half maximum is $\Delta \tau=100 \mathrm{psec}$. The constant $P_{0}$ can be related to the laser's operating point with respect to the oscillation threshold. Oscillation was achieved with a mirror transmission of $46 \%$, but not with a transmission of $65 \%$. Hence, the limiting threshold parameter for the reported experiments must have been somewhere between unity and at most $r \simeq 65 / 46=1.4$. The pump constant $P_{0}$ may be chosen in such a way that Eqs. $(62)-(64)$ and (66) produce the desired value of $r_{0}$. This threshold parameter $r_{0}$ can be identified with the maximum value of the ratio

$$
\int_{0}^{1} Q x d x / A
$$

whose maximum is reached near the end of the pump pulse, when the population depletion term $2 Q A x$ is set to 0 in Eq. (62). This procedure of determining $P_{0}$ for a given value of $r_{0}$ has been carried out numerically as a preliminary step in the computation of the field and population pulsations. For the calculations described below, $P_{0}$ has been chosen to correspond to the reasonable parameter value $r_{0}=1.3$.

\section{RESULTS}

A theoretical model has now been described which should govern the parameters of the mode-locked pulsations in a synchronously pumped dye laser oscillator. The numerical constants needed to apply this model to a rhodamine $6 \mathrm{G}$ dye laser have also been discussed. In this section, the theoretical equations are solved in general and compared to the experimental autocorrelation data that has been described previously. Various limits and approximations are also discussed.

\section{A. General solutions}

A typical solution of Eqs. (62)-(64) and (66) is given in Fig. 4. The parameter values used in this solution are (as discussed previously) $\tau_{2}=5 \times 10^{-9} \mathrm{sec}, \tau_{1}=1 \times 10^{-12} \mathrm{sec}$, $T_{s}=5 \times 10^{-14} \mathrm{sec}, t_{c}=4 \times 10^{-9} \mathrm{sec}$, and $L=1.8 \mathrm{~m}$. The pump $P$ is adjusted to yield $r_{0}=1.3$, and a weak spontaneous emission noise source proportional to the upper state population has been introduced to start the numerical solutions. In addition, the cavity length mismatch has been chosen as $\Delta L=1 \times 10^{-5} \mathrm{~m}$, and the initial values of the populations, polarization, and field are all zero.

For compactness, several different results are summarized in Fig. 4. The curve labeled $f$ is the Gaussian pump function from Eq. (67). The full width at half maximum of this function is $\Delta \tau=100 \mathrm{psec}$, and the scale is chosen arbitrarily so that the pump maximum has a value of 500 on the intensity $I=A^{2}$ scale. The time scale is arbitrarily set so that the origin occurs at the center of the pump pulse. The curve labeled $r$ represents the time-dependent threshold parameter defined above. As soon as $r$ exceeds unity, there is a net gain in the electric field, and for this example, $r$ would have ap- 


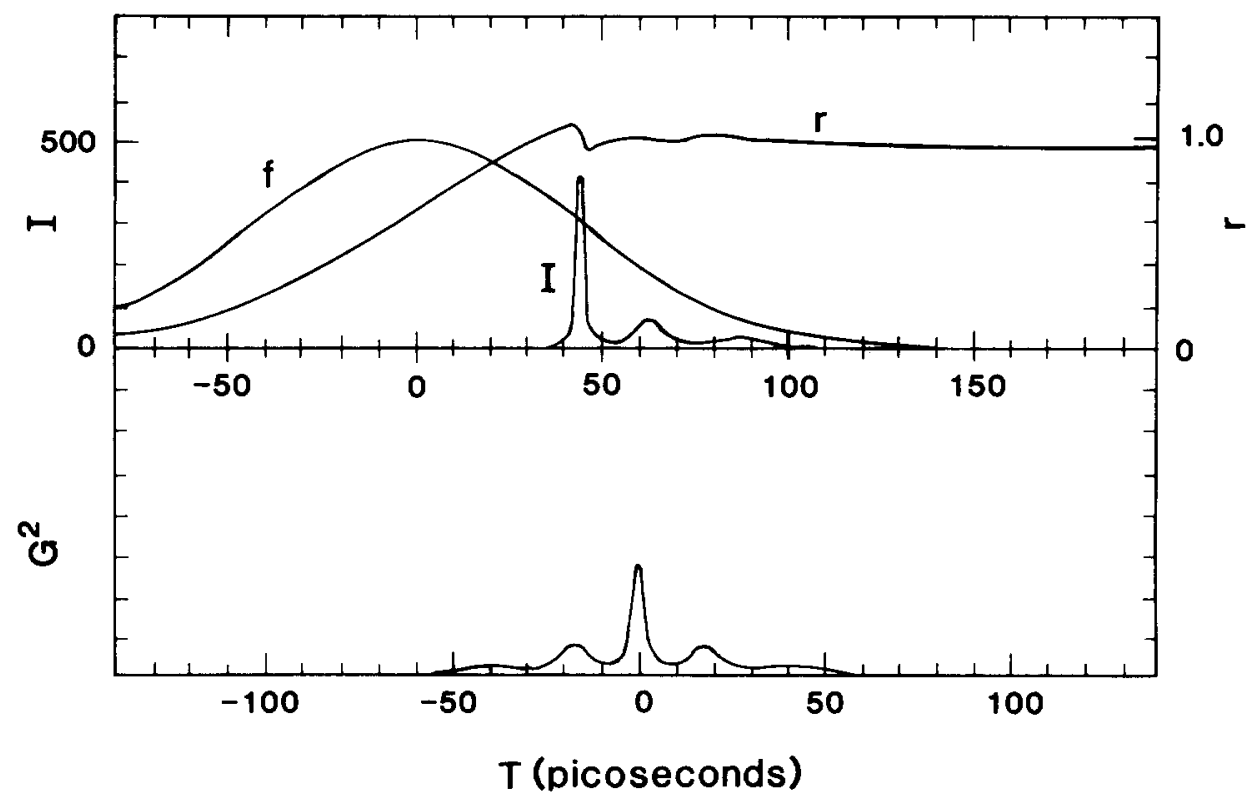

FIG. 4. Computed curves of the pump function $f$, the thereshold parameter $r$, the intensity $I=A^{2}$, and the intensity autocorrelation $G^{2}$ for a detuning of $\Delta L=10 \mu \mathrm{m}$. Parameters used in deriving these results agree as closely as possible with the actual experimental values.

proached the limiting value $r_{0}=1.3$. However, shortly after $r$ becomes greater than unity, a saturating intensity pulse develops which abruptly reduces the value of $r$. There follows a period of damped ringing until the pump pulse ends, and the threshold parameter is left at a value close to $r=1$. Although it is not shown clearly in the figure, the threshold parameter continuous to slowly decay, with a time constant given approximately by $\tau_{2}=5 \times 10^{-9} \mathrm{sec}$

The qualitative behavior of $f, r$, and $I$ as illustrated in Fig. 4 has been recognized for several years, and this behavior can be understood in terms of very simple theoretical models. However, no previous model has been successful at quantitatively predicting the details of the pulsation process. The present analysis includes a characterization of several physical processes which are usually neglected, and for the first time, it becomes meaningful to attempt a rigorous comparison of theoretical and experimental pulsation waveforms.

In principle at least, it is possible to directly observe intensity waveforms of the sort shown in Fig. 4. Streak cameras with picosecond resolution are commercially available, and such devices have been widely used to study the ultrashort pulses from mode-locked dye lasers. ${ }^{20}$ It is much more conventional and inexpensive, however, to use nonlinear optical methods to obtain the autocorrelation of the intensity pulses

$$
G^{2}\left(\tau^{\prime}\right)=\int_{-\infty}^{\infty} I(\tau) I\left(\tau+\tau^{\prime}\right) d \tau
$$

The lower trace in Fig. 4 is the computed autocorrelation of the intensity pulse shown above it.

The curves shown in Fig. 4 correspond to a length detuning of $10 \mu \mathrm{m}$. Many similar compuations have been carried out, and a series of intensity autocorrelations for various length detunings is shown in Fig. 5. With a detuning of greater than about $50 \mu \mathrm{m}$, the output consists of a single pulse which gradually broadens and weakens with increased detuning. As the cavity length approaches exact synchrony, increasing numbers of secondary pulses are in evidence, and the field retains a substantial value between these pulse echoes.

The theoretical curves shown in Fig. 5 may be compared directly to the experimental curves of Fig. 3. It can be observed that even in many detailed features, the two sets of

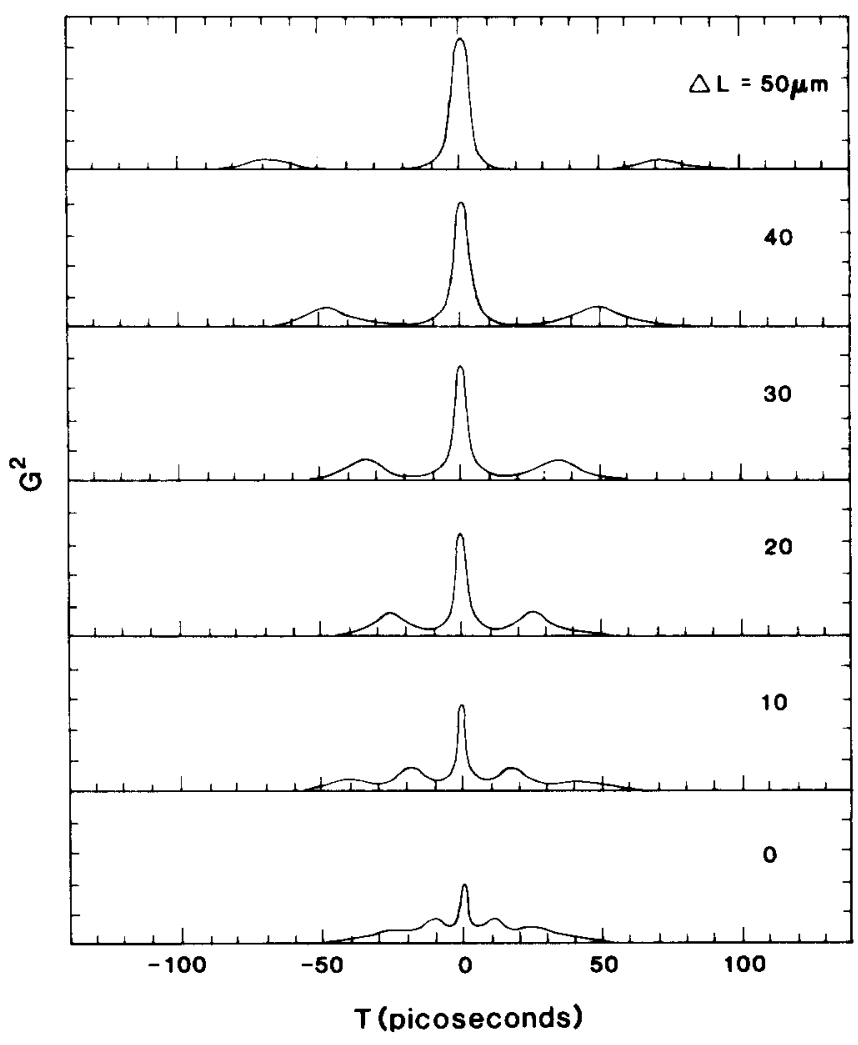

FIG. 5. Intensity autocorrelations for various values of detuning based on the general theoretical model given in Eqs. $(62)-(64)$ and (66). These results agree with the experimental curves in Fig. 3 if the relative detunings are changed to absolute detunings by subtracting $455 \mu \mathrm{m}$ 
curves are in quantitative agreement if the relative detuning lengths in Fig. 3 are changed to absolute detuning lengths by subtracting $455 \mu \mathrm{m}$. The slight remaining discrepancies may be due in part to limited resolution of the experimental results and the neglect in the calculations of the coherence spike. For small detunings, the radiation makes many round trips before ceasing to be synchronous with the gain pulse, so spontaneous emission noise effects should be relatively unimportant. For large detunings, however, experiments and "noise-burst" calculations show a conspicuous coherence spike sitting atop the intensity autocorrelation curves. ${ }^{21,22}$

A few additional comments on the numerical calculations may be appropriate. Figure 5 includes numerical data for the case of zero detuning $\Delta L=0$. However, it is clear from Eq. (66) that for $\Delta L=0$, the right-hand side of the equation becomes infinite. Thus, in the numerical solutions of Eqs. (62)-(64) and (66), one must never let the detuning become exactly zero, and it is necessary to obtain the $\Delta L=0$ behavior using very small detunings. Unfortunately, numerical solutions for small values of $\Delta L$ are very slow and a better approach is to transform the initial equations. If $\Delta L$ in Eq. (66) goes to zero and the rate of change of the field is to remain finite, it is clear that the field amplitude must at all times be given by

$$
A=\int_{0}^{1} Q x d x
$$

The set of Eqs. (62)-(64) and (68) can be integrated without difficulty, and this procedure has been used in all $\Delta L=0$ integrations.

The actual time integrals have been carried out using a second-order Runge-Kutta method, while the angular integrals have been performed using Simpson's rule. ${ }^{23}$ This general approach is similar to that used in the study of coherent transients in gas lasers, where the inhomogeneous broadening integrals are roughly analogous to the present angular distribution integrals. ${ }^{24}$ Time steps $d \tau$ and angle steps $d x$ have been varied extensively to ensure that none of the reported pulsation characteristics are artifacts of the numerical procedures. For most calculations, step sizes of $d \tau=10^{-14} \mathrm{sec}$ and $d x=0.1$ have proved to be adequately small.

Another aspect of these calculations may also merit a brief comment. Technically, the initial values of the populations, polarization, and field just prior to the arrival of a pump pulse should consist of whatever remains of these quantities from the previous pulse, whereas in the present calculation, these initial values have all been set to 0 . That the initial values of $A$ and $Q$ can be regarded as 0 follows from the extremely short decay times of these quanties in Eqs. (64) and (66). It is not quite so obvious that the initial value of the upper state population can be regarded as 0 . The lifetime of the upper state has been taken as $5 \mathrm{nsec}$, so this population should decay in a round trip time $t_{\mathrm{rt}}=2 L_{\text {dye }} / v_{s}=12 \mathrm{nsec}$ by the factor $\exp (-12 / 5)=0.09$. However, the effective rotational diffusion lifetime for rhodamine $6 \mathrm{G}$ in ethylene glycol is about $2.1 \mathrm{nsec}^{25}$ so most of the remaining upper state molecules will have rotated into relatively ineffective angular positions. Thus, neglect of the initial population of the upper state is quite well justified and leads to errors which should be very small compared, for example, to those arising from uncertainties in the pump pulse waveform which puts atoms into the upper state. An important advantage of this type of formulation is that it is only necessary to integrate over a single pump pulse to obtain the output pulse shape.

The close agreement between the complicated experimental data in Fig. 3 and the theoretical curves given in Fig. 5 is evident. While this agreement lends credibility to the theoretical model, it is natural to reexamine the number of adjustable parameters involved. The model obviously contains many parameters, but for the most part, they are not very adjustable. All of the parameters of the dye itself are reasonably well known (at least within a factor of 2 ), and an attempt was made here to adopt the most popular values for $\tau_{2}, \tau_{1}$, and $T_{s}$. The threshold parameter $r_{0}$ was not precisely specified in the experimental study, and in our numerical modeling, the cavity lifetime $t_{c}$ has been varied over reasonable values to obtain the indicated fit. These are the only parameters that have been adjusted in obtaining our theoretical results, and no more rigorous test of the model seems to be possible using existing experimental data.

\section{B. Unidirectional molecular distribution}

The model described here includes several complicating features not found in most previous studies of synchronously pumped mode-locked dye lasers. However, the agreement of the model with experimental data does not by itself ensure that all of these complications are really necessary. It could turn out that equally good agreement would be obtainable with a simplified version of the model, and considerable savings in computer time. To test this possibility, several reasonable approximations have been explored.

One of the most time-consuming features of the analysis is the inclusion of an isotropic molecular distribution. As a consequence of this distribution, it is necessary to represent each of the population and polarization components by a large number of variables coresponding to the various possible molecular orientations. However, with parallel pump and signal polarizations and slow rotational relaxation, it seems possible that most of the dye molecules would contribute little to the absorption and amplification processes, and only the molecules that are roughly parallel to the fields would need to be considered. A unidirectional orientational distribution can be written $n(\theta, \phi)=N \delta\left(\theta-\theta_{0}, \phi-\phi_{0}\right)$, where $\delta$ is a normalized delta function, and $\theta_{0}$ and $\phi_{0}$ indicate the alignment direction. If this distribution is substituted into Eqs. (45) $-(48)$ with $\theta_{0}=0$, the results have the same form as Eqs. (49)-(52), except that $x$ is replaced by unity in Eqs. (49)-(51) and the integral is simply replaced by $n_{s}^{\prime}$ in Eq. (52). Proceeding as before, one finds that the final set of Eqs. (62)-(64) and (66) are replaced by the unidirectional set

$$
\begin{aligned}
\frac{d D}{d \tau}= & -\frac{1}{\tau_{2}}\left[\left(1+\frac{\tau_{2}}{2 \tau_{1}}\right) D,\right. \\
& \left.+\left(1-\frac{\tau_{2}}{2 \tau_{1}}\right) M+2 Q A-P\right], \\
\frac{d M}{d \tau}= & -\frac{1}{\tau_{2}}\left(-\frac{\tau_{2}}{2 \tau_{1}} D+\frac{\tau_{2}}{2 \tau_{1}} M-P\right),
\end{aligned}
$$




$$
\begin{aligned}
& \frac{d Q}{d \tau}=-\frac{1}{T_{s}}(Q-A D), \\
& \frac{d A}{d \tau}=-\frac{L}{2 t_{c} \Delta L}(A-Q) .
\end{aligned}
$$

Equations (69)-(72) can be integrated much more easily than the previous set, and a Runge-Kutta derivation of the intensity pulses is possible in principle with some programmable pocket calculators. A set of intensity autocorrelation solutions of these equations is given in Fig. 6, and the parameter values used in obtaining these results are exactly the same as used previously in obtaining the curves given in Fig. 5. The case $\Delta L=0$ again requires special treatment, and from Eq. (72) one obtains the simple result $A=Q$. With this substitution, Eqs. $(69)-(71)$ can be written

$$
\begin{aligned}
\frac{d D}{d \tau}= & -\frac{1}{\tau_{2}}\left[\left(1+\frac{\tau_{2}}{2 \tau_{1}}\right) D\right. \\
& \left.+\left(1-\frac{\tau_{2}}{2 \tau_{1}}\right) M+2 A^{2}-P\right], \\
\frac{d M}{d \tau}= & -\frac{1}{\tau_{2}}\left(-\frac{\tau_{2}}{2 \tau_{1}} D+\frac{\tau_{2}}{2 \tau_{1}} M-P\right), \\
\frac{d A}{d \tau}= & -\frac{A}{T_{s}}(1-D) .
\end{aligned}
$$

These equations were used to obtain the $\Delta L=0$ curve in Fig. 6.

The intensity pulses for the unidirectional distribution

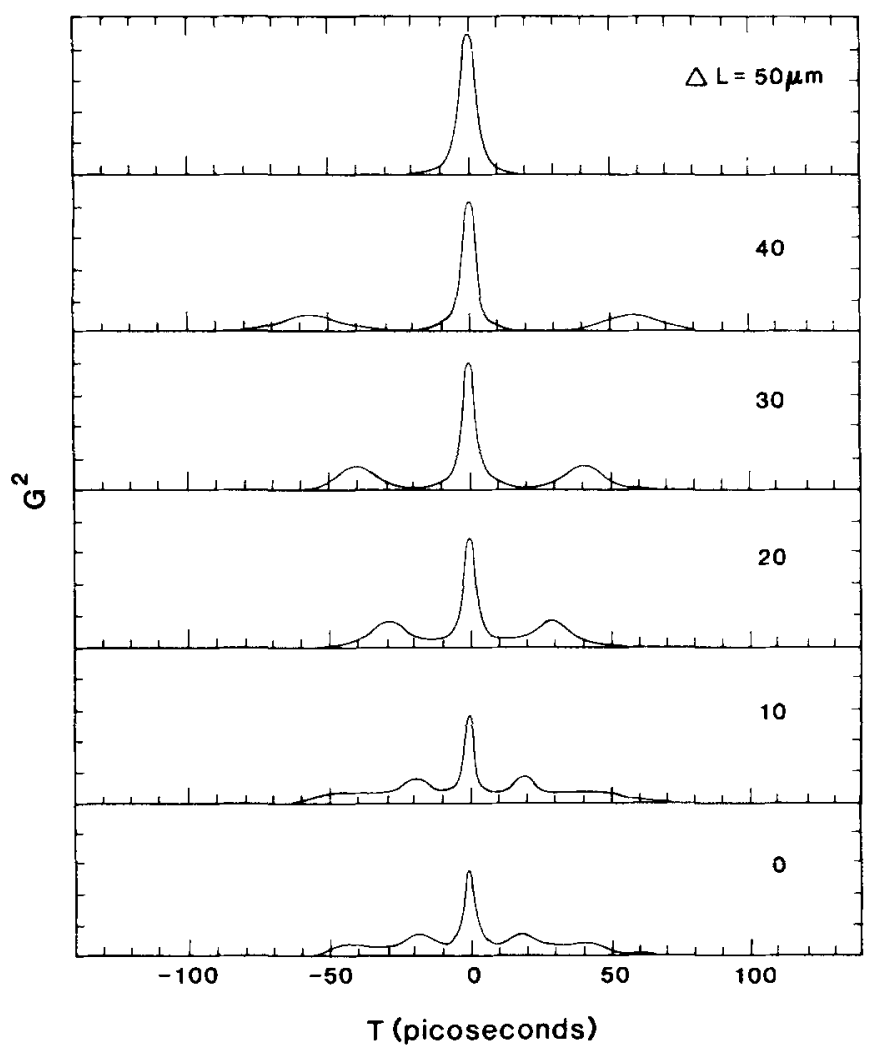

FIG. 6. Intensity autocorrelations for various values of the detuning assuming an isotropic molecular distribution. The vertical sensitivity here is twice as great as in Fig. 5, i.e., the intensity pulses are somewhat smaller in this approximation. are, in general, quite similar to those obtained in the more exact treatment. One difference is that the pulse amplitudes are slightly smaller in the approximate case, and this amplitude discrepancy leads to about a factor of 2 difference in the autocorrelation amplitudes. For convenience of comparison, the vertical scale sensitivity in Fig. 6 is exactly twice as great as that in Fig. 5. Except for this amplitude decrease and a reduced echo frequency, the unidirectional approximation should be adequate for most qualitative studies. For quantitative interpretations or predictions, the exact isotropic molecular distrbution must be included.

\section{Short coherence time}

Another complication of the present analysis is that it includes a finite coherence time for the molecular wave functions. Stated differently, this model allows the polarization to depend on the past history of the field and populations. This is in contrast to rate equation studies, in which the polarization is an instantaneous function of the field and populations. The importance of retaining a finite coherence time can be investigated by recomputing the laser behavior in the $\operatorname{limit} T_{s}=0$. It has already been shown that the assumption of a unidirectional molecular distribution does not effect the qualitative laser behavior, and for simplicity, the unidirectional assumption is retained in the following investigation. Thus the starting equations here are Eqs. (69)-(72).

In the rate equation limit $T_{s}=0$, Eq. (71) has the solution $Q=A D$. With this substitution, Eqs. (69), (70), and (72) become

$$
\begin{aligned}
& \frac{d D}{d \tau}=-\frac{1}{\tau_{2}}\left[\left(1+\frac{\tau_{2}}{2 \tau_{1}}\right) D\right. \\
&\left.+\left(1-\frac{\tau_{2}}{2 \tau_{1}}\right) M+2 A^{2} D-P\right], \\
& \frac{d M}{d \tau}=-\frac{1}{\tau_{2}}\left(-\frac{\tau_{2}}{2 \tau_{1}} D+\frac{\tau_{2}}{2 \tau_{1}} M-P\right), \\
& \frac{d A}{d \tau}=-\frac{L A}{2 t_{c} \Delta L}(1-D) .
\end{aligned}
$$

The intensity autocorrelations implied by these equations are plotted in Fig. 7, using the same parameter values employed previously in plotting Fig. 6 (except that in Fig. 7, $T_{s}=0$, and the vertical sensitivity is half as great). It is evident from a comparison of Figs. 6 and 7 that some qualitative features of the pulsations are similar whether or not the finite coherence time is explicity included. For quantitative calculations, however, the rate equation model $\left(T_{s}=0\right)$ is seriously deficient, and the discrepancy is greatest for small amounts of detuning.

The autocorrelation in the case $\Delta L=0$ is a smooth curve without ripples, and the reason for this can be readily understood. The effective gain in (78) becomes so large for small $\Delta L$ that the field rises instantaneously to saturate the population difference at the threshold value $D=1$. The intensity then varies gradually in such a way that the population difference remains at $D=1$ until the end of the pump pulse, and this general behavior has been discussed in a previous rate equation treatment. ${ }^{6}$ This ability of the laser am- 


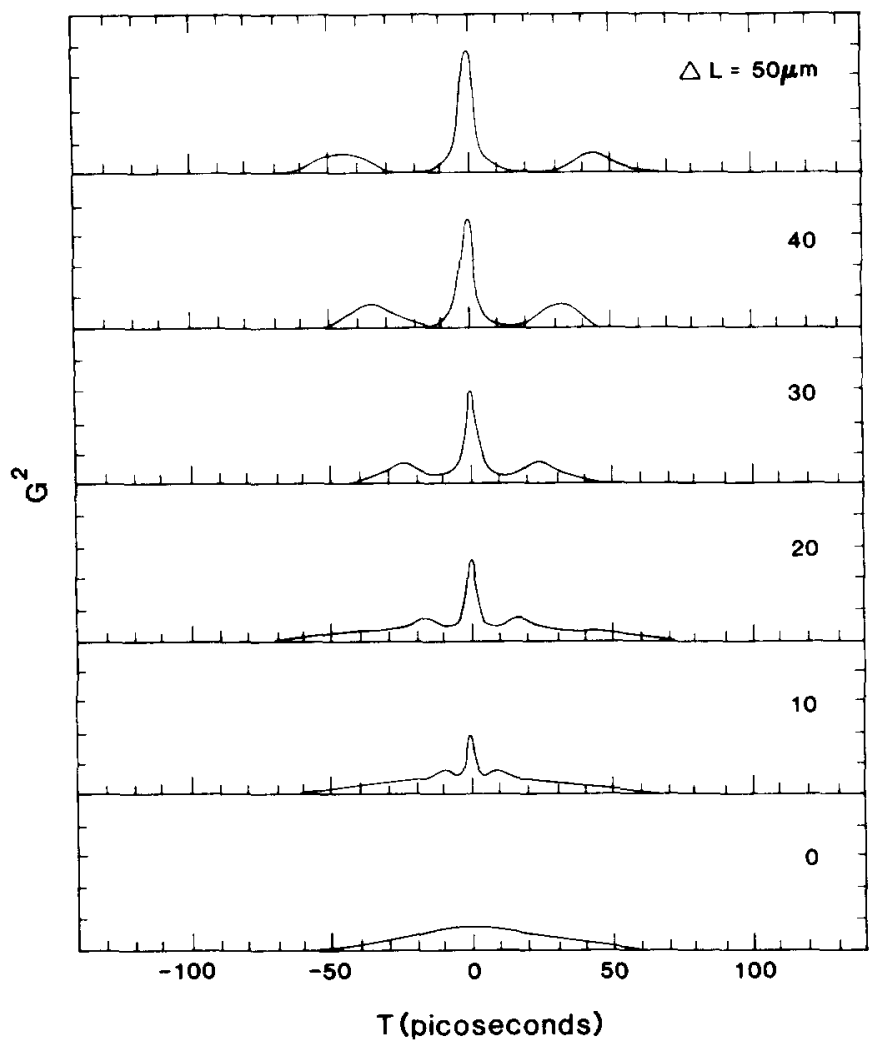

FIG. 7. Intensity autocorrelations assuming that coherence effects can be neglected $\left(T_{s}=0\right)$. The vertical sensitivity is the same as in Fig. 5 .

plifier to support a pulse with zero rise time is physically unrealistic, and its occurrence here illustrates one of the principal limitations of rate equation treatments of pulse propagation. Physically, no amplifier can support a zero rise time pulse, because the polarization cannot respond instantaneously to the field.

\section{Fast vibrational decay}

The general formalism developed here has included a characterization of vibrational decay in the lower laser state. As a final step in these studies, the importance of including this finite vibrational relaxation time will be examined by setting the relaxation time to 0 and examining the validity of the remaining model. Again, it is sufficient to use Eqs. (69)(72) as a starting point.

By adding Eqs. (69) and (70) and dividing by 2 , one finds that the normalized population is governed by

$$
\frac{d}{d \tau}\left(\frac{D+M}{2}\right)=-\frac{1}{\tau_{2}}\left[\left(\frac{D+M}{2}\right)+Q A-P\right] \text {. }
$$

But in the limit of fast vibrational relaxation, the lower laser state is empty and the population difference $D$ equals the population sum $M$. Therefore, Eq. (79) can be written more simply as

$$
\frac{d D}{d \tau}=-\frac{1}{\tau_{2}}(D+Q A-P)
$$

The remaining equations governing this model are Eqs. (71) and (72), and their forms are unchanged in this limit.

Equation (71), (72), and (80) have been solved numeri- cally, and a set of intensity autocorrelations is displayed in Fig. 8. As in the approximations considered previously, the autocorrelations still bear some qualitative resemblance to the more exact model represented by Fig. 6 (the vertical scale is the same this time). But again there are major quantitative discrepancies, and the greatest differences occur for small levels of detuning. That significant differences should occur is reasonably obvious from the fact that the vibrational relaxation time $\left(\tau_{1} \simeq 1 \mathrm{psec}\right)$ is of the same order as the pulse length. These results show that quantitative modeling of the synchronously pumped mode-locked dye laser is not possible unless vibrational relaxation is included.

\section{CONCLUSION}

A theoretical model has been developed for characterizing the very short pulses obained in synchronously pumped mode-locked dye lasers. The pulses predicted by this model are in good agreement with experimental results that have been published, and the theoretical pulses have been compared in detail with a particular set of pulsation waveforms. A wide range of pulse characteristics have been fitted varying only a small number of parameters in the model, and it is possible that in a better characterized experiment, no adjustments would be required. No previous model has been successful in quantitatively predicting the actual pulsation waveforms.

In spite of the good agreement between theory and experiments, the model described here is obviously not yet complete. There are several other considerations that could

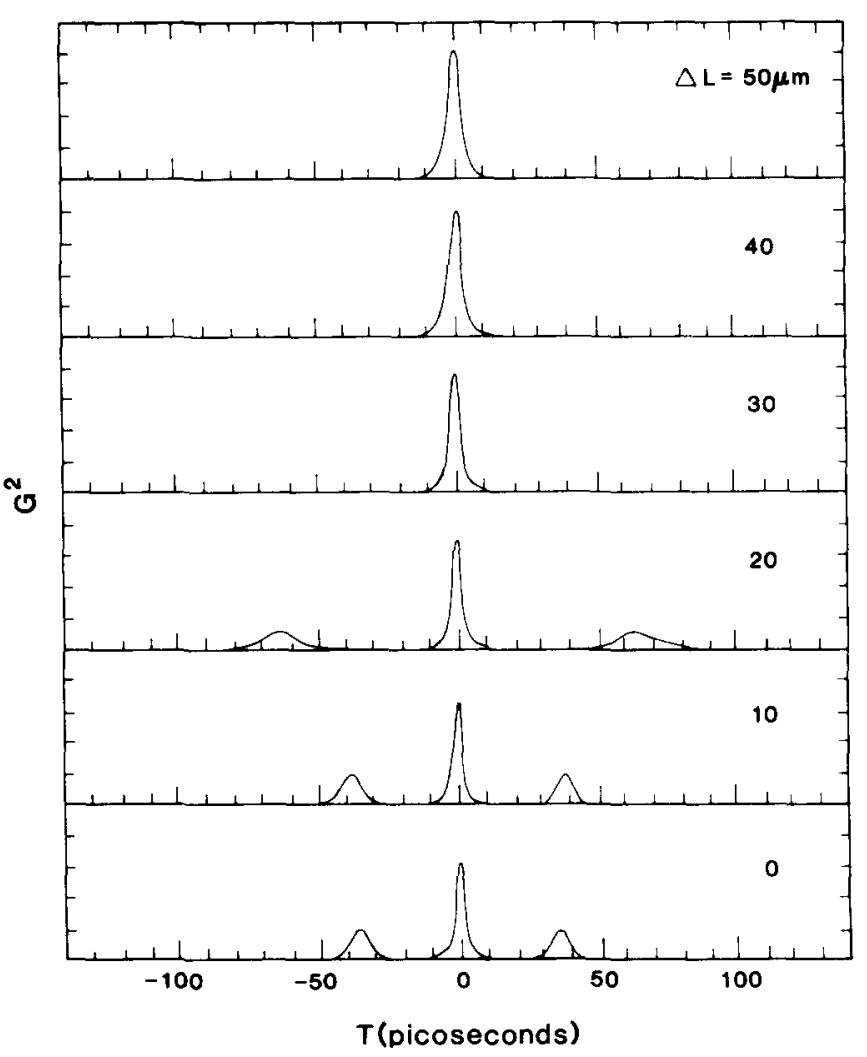

FIG. 8. Intensity autocorrelations assuming that vibrational relaxation can be neglected $\left(\tau_{1}=0\right)$. The vertical sensitivity is the same as in Fig. 6. 
lead to modest or even substantial improvements. A rigorous model of noise is needed to predict the form of the coherence spike observed at large detunings. Depending on the details of any frequency selective cavity elements, it might be important to explicitly account for the resulting dispersion effects independent of the dispersion of the laser molecules. The difference between standing-wave and traveling-wave dye lasers should be investigated, including spatial holeburning and dye-placement effects. The single pass gain may be quite large, and the small-gain approximation needs justification. The beam is usually a Gaussian rather than a uniform plane wave. These and other approximations deserve a more careful treatment. One purpose here has been to develop the simplest possible model which leads to reasonable agreement with experiments, and further studies may lead to significant enhancements of the model.

Specific features of the present model which have not been included in most previous studies include an isotropic orientational distribution of the dye molecules, a nonzero coherence time, and a nonzero vibrational relaxation time. An attempt has been made to use the best available data for all of the laser parameters, and the numerical studies have shown that good agreement is possible only when all of the features mentioned above are included. Thus, it cannot be claimed here with conviction that no significant effects are missing from the model. It can be claimed, however, that any accurate model will have to explicitly include the isostropic molecular distribution, the finite coherence time, and vibrational relaxation.

There are several applications that can be visualized for a model of the synchronously pumped mode-locked dye laser. The most obvious possibility is that one can predict with some confidence the detailed output waveforms of a modelocked rhodamine $6 \mathrm{G}$ laser before the laser is built. As data on other dyes becomes more complete, predictions regarding other picosecond dye lasers will be possible as well, and such predictions might lead to savings of time and money. Also, it might be possible to use the output pulsation waveforms as a diagnostic of the laser dye itself. The very short coherence and vibrational relaxation times are difficult to measure directly, but numerical calculations show that the precise values of these times sensitively affect the shapes of the modelocked pulses. Hence, numerical curve fitting may be an effective means for determining these lifetimes from experimental mode-locking data.

Finally, it may be noted that most experiments with mode-locked pulses measure the intensity autocorrelations rather than the actual intensity pulses, and there is always a degree of uncertainty in determining a pulse shape from its autocorrelation. However, for most applications, it is the original pulse shape that one would like to know, and this pulse shape can be obtained with the aid of a theoretical model. If a model has been developed which explains an observed autocorrelation, the intensity pulse is at once fully characterized. Thus, the agreement between the $\Delta L=10$ $\mu \mathrm{m}$ autocorrelation in Fig. 4 and the curve labelled 465 in Fig. 3 suggests that the actual intensity pulse corresponding to the experiment looks like the intensity curve given in the top portion of Fig. 4.

\section{ACKNOWLEDGMENTS}

The author expresses his appreciation to J. Patel, L. M. Davis, J. D. Harvey, and other members of the Physics Department at the University of Auckland for valuable discussions and hospitality during his sabbatical visit in which much of this project was carried out.

'A. Dienes, E. P. Ippen, and C. V. Shank, in Topics in Applied Physics (Springer, New York, 1973), Vol. 1, p. 137.

${ }^{2}$ C. K. Chan and S. O. Sari, Appl. Phys. Lett. 25, 403 (1974).

${ }^{3}$ J. M. Harris, R. W. Chrisman, and F. E. Lytle, Appl. Phys. Lett. 26, 16 (1975).

${ }^{4}$ H. Mahr and M. D. Hirsch, Opt. Commun. 13, 96 (1975).

${ }^{5}$ Z. A. Yasa and O. Teschke, Opt. Commun. 15, 169 (1975).

${ }^{6}$ A. Scavennec, Opt. Commun. 17, $14(1976)$.

${ }^{7}$ N. J. Frigo, T. Daly, and H. Mahr, IEEE J. Quantum Electron. QE-13, 101 (1977).

${ }^{8}$ C. P. Ausschnitt, R. K. Jain, and J. P. Heritage, IEEE J. Quantum Electron. QE-15, 912 (1979)

${ }^{9} \mathrm{~J}$. Patel, 1981 (private communication).

${ }^{10}$ W. E. Lamb, Jr., Phys. Rev. A 134, 1429 (1964).

${ }^{1}$ K. C. Reyzer and L. W. Casperson, J. Appl. Phys. 51, 6075 (1980).

${ }^{12}$ K. C. Reyzer and L. W. Casperson, J. Appl. Phys. 51, 6083 (1980).

${ }^{13} \mathrm{D}$. Ricard and J. Ducuing, IEEE J. Quantum Electron. QE-10, 745 (1974).

${ }^{14}$ D. Ricard and J. Ducuing, J. Chem. Phys. 62, 3616 (1975).

${ }^{15}$ A. Penzkofer, W. Falkenstein, and W. Kaiser, Chem. Phys. Lett. 44, 82 (1976).

${ }^{16}$ T. Yajima, H. Souma, and Y. Ishida, Phys. Rev. A 17, $324(1978)$.

${ }^{17}$ M. M. Malley and G. Mourou, Opt. Commun. 10, 323 (1974).

${ }^{18}$ B. K. Garside and T. K. Lim, Opt. Commun. 8, 297 (1973)

${ }^{19}$ B. K. Garside and T. K. Lim, Opt. Commun. 12, 240 (1974).

${ }^{20}$ E. P. Ippen and C. V. Shank, Top. Appl. Phys. 18, 83 (1977).

${ }^{21}$ A. E. Siegman and D. J. Kuizenga, Opto-electronics 6, 43 (1974).

${ }^{22}$ D. B. McDonald, D. Waldeck, and G. R. Fleming, Opt. Commun. 34, 127 (1980).

${ }^{23}$ Handbook of Mathematical Functions, (Nat. Bur. Stand. Appl. Math. Ser. 55) edited by M. Abramowitz and I. A. Stegun (Washington, D.C., 1970), p. 897.

${ }^{24}$ L. W. Casperson, IEEE J. Quantum Electron. QE-14, 756 (1978).

${ }^{25}$ T. J. Chuang and K. B. Eisenthal, Chem. Phys. Lett. 11, 368 (1971). 